\title{
A Probe Into Chinese University Students’ English Lexical Ambiguity
}

\author{
Xiaochun Zhang ${ }^{1}$ \\ ${ }^{1}$ School of Foreign Languages, Leshan Normal University, Leshan, China \\ Correspondence: Xicochun Zhang, School of Foreign Languages, Leshan Normal University, 778\#, Binhe Road, \\ Shizhong District, Leshan, Sichuan Province, China.
}

Received: July 30, 2019 Accepted: September 9, 2019 Online Published: September 11, 2019

doi: 10.5539/elt.v12n10p55 URL: https://doi.org/10.5539/elt.v12n10p55

\begin{abstract}
Disagreements arise on the differences of semantic processing of different ambiguous words in the perspective of psycholinguistics. This paper compares the differences of the semantic processing of different types of ambiguous words of Chinese English learners by using a multiple semantic priming experiment with short. The results demonstrate the advantage in semantic processing of words of homonymy of Chinese English Learners in the multiple semantic priming experiment, but the advantage in semantic processing of words of polysemy does not always take place, as it is relevant to learners' English levels and words' meaning frequency. The effect of semantic processing of polysemous words is greater than that of synonymous words.
\end{abstract}

Keywords: semantic processing, lexical ambiguity, homonymy, polysemy

\section{Introduction}

Lexical ambiguity is a common phenomenon in language that one word shape corresponds to several different meanings. For example, the meaning of "bank" is the side of a river in the sentence "He sat on the bank of the river and watched the currents", while its meaning is an organization that provides various financial services in the sentence "He cashed the check at the bank. The meaning of the word "door" is a piece of wood or glass that is opened and closed so that people can get in and out of a room or building in the sentence "Mary painted the door", while its meaning is the space when a door is open in the sentence "Mary walked through the door" (Klepousniotou, 2007: 19). Although both "bank" and "door" are the ambiguous words which have two or more meanings, there are different relationships between their meanings. Two different meanings of "bank" represent two unrelated concepts. However, two meanings of the word "door" have a core meaning of entrance and exit. According to the semantic relationship between the meanings, the ambiguous words are classified as homonym and polysemy by the linguistics (Weinreich, 1964; Lyons, 1977; Cruse, 1986). There is no semantic correlation between the two meanings of a homonym, such as bank. Nevertheless, there is a semantic correlation between the two meanings of a polysemy, such as door. Are these two types of ambiguous words the same as the mental representations?

The mental representation and processing of ambiguous words is one of the main research questions in the field of psycholinguistics. Usually, the theory of connectionism is adopted by psycholinguistics to explain the semantic processing and representation of ambiguous words (Hino et al., 2002; Rodd et al., 2004; Armstrong, \& Plaut, 2011). According to the theory of connectionism, concepts are represented by activation patterns of a set of connection-weighted units distributed in a semantic network. A single unit cannot represent concepts locally, but can be represented in different concepts, which makes the activation patterns of some concepts similar. Psycholinguistics consider the concept with similar activation patterns as semantically relevant concept. It can be seen that the overlapping semantic feature is a main feature of semantically related concepts. The degree of correlation between concepts is determined by the number of overlapping semantic features between concepts and their state in conceptual representation. The overlapping semantic features determine the mutual promotion between related concepts (McRae et al., 1997: 103). Therefore, for different types of ambiguous words, the speed of semantic processing is a kind of way or method to judge their mental representations and differences of processing. If the meanings of homonym are related to each other, while the meanings of polysemy are not related, then the semantic processing speed of polysemy should be faster than that of homonym.

Psycholinguistics began to pay attention to the differences between polysemy and homonym since 1997 (Azuma, 1997). It is generally agreed that the meanings of homonym are not semantically related and represented by 
independent terms. However, it is still controversial that whether the meanings of polysemy are semantically related (Klein \& Murphy, 2001; Rodd et al., 2002; Klepousniotou, 2007). Comparing the unambiguous words with the processing speed of homonyms and polysemous words through lexical judgement experiment, some researchers (Rodd et al., 2002; Beretta et al., 2005; Armstrong \& Plaut, 2008, 2011) find that the processing speed of polysemous words is faster than that of unambiguous words, while processing speed of homonyms is slower than that of unambiguous words. According to the theory of connectionism, the researchers believe that the processing advantaged effect of polysemous words is the effect with meaningful correlation, whereas the disadvantaged effect of homonyms is the inhibiting effect between unrelated meanings. Therefore, they consider that there are differences in representation and processing between the two types of ambiguous words. Nonetheless, other researchers (Hino et al., 2002; Hino et al., 2010) are skeptical about the use of lexical judgement experiment to demonstrate semantic processing effect. They argue that the advantaged effect of polysemy processing in lexical judgement task is the result of mapping from semantic layer to orthography layer. Because the lexical processing system in the mental lexicon is composed of orthography, phonetics and semantics are connected with each other. Vocabulary recognition is a process from orthography layer to semantic layer or from semantic layer to orthography layer, which is the process from bottom to top or from top to bottom respectively. The advantaged effect of polysemy is caused by a large amount of feedback mapping from semantic layer to orthography layer when multiple meanings are activated. Different from the experimental methods of the above researchers, some researchers find that there is no mutual promotion effect between the meanings of polysemous words through adopting the method of word meaning judgement. Therefore, they believe that the meaning representation of polysemous words is the same as that of homonyms. It can be seen that the experimental method is an important factor that leads to the inconsistencies in the research conclusions about the processing and representation differences of different types of ambiguous words.

The multiple semantic priming experiment is an important experimental method for psycholinguistic research. It includes two or more priming stimulus (such as kidney-organ-piano). The multiple semantic priming effects refers to the promotion effect of two successively presented priming words on the subsequent semantically related target words. The multiple semantic priming experiment is mostly used to study the complex structure of semantic networks and the semantic processing mechanism of online words. In addition, masking and short SOA (The time between the priming stimulus presentation and the target stimulus presentation) are two ways to improve automatic processing (Needly, 1977; de Groot, 1984). Automatic process refers to the process of rapid semantic processing in the unconscious and unmindful state (McNamara, 2005).

This study employs the lexical judgment task in a masked multiple semantic priming experiment with short SOA to investigate the semantic processing differences of different types of ambiguous words among Chinese English learners. Research hypothesis: Different levels of Chinese English learners have various semantic processing effects on different types of ambiguous words in the multiple semantic priming experiment. The semantic processing speed of polysemous words is faster than that of control words, and the semantic processing speed of homonymous words is slower than that of control words.

\section{Experiment}

\subsection{Participants}

70 undergraduate students majoring in English in a provincial college were recruited for this experiment, including 34 senior students and 36 freshmen. In the experiment, senior students were defined as high-level participants and freshmen as low-level participants according to their grade. All the participants had normal vision or corrected vision. In order to avoid repeated occurrence of priming words, the experiment was divided into two experimental groups according to the frequency of the use of ambiguous words: primary meaning and secondary meaning. High-level and low-level participants were randomly divided into two groups and conducted different meaning-biased priming experiments. Each experimental group consisted of 17 high-level participants and 18 low-level participants. Chinese is the first language of all the participants, and English is their second language. Before participating in this experiment, all the participants had conducted subjective assessment of basic English skills and vocabulary level 5 test of the Nation (1993). The results of subjective assessment of basic English skills and vocabulary level test are described in table 1.

In this study, SPSS 22.0 was conducted to analyze the variance of intra-group and inter-group on the results of autonomous assessment of basic English skills and vocabulary level test. The results showed that there was no significant difference in the results of autonomous assessment between high and low-level participants, while there was significant difference in the results of vocabulary test of level $5(\mathrm{p}<0.05)$. There was no significant difference between participants in primary and secondary meaning-biased priming experiments. 
Table 1. Description of the results of basic skills and vocabulary level test

\begin{tabular}{lllll}
\hline & & \multicolumn{2}{c}{ High-level } & Low-level \\
& Primary (M) & Secondary (M) & Primary (M) & Secondary (M) \\
\hline Age & 21 & 21 & 18 & 18 \\
$\begin{array}{l}\text { Duration of English } \\
\text { learning (years) }\end{array}$ & 9.76 & 10.10 & 9.55 & 8.75 \\
Speaking & 5.76 & & & \\
Reading & 6.56 & 6.55 & 6.30 & 5.20 \\
Listening & 6.18 & 6.68 & 6.48 & 5.75 \\
Writing & 6.30 & 6.30 & 6.60 & 5.60 \\
2000 words & 28.00 & 6.05 & 6.25 & 5.70 \\
3000 words & 27.71 & 28.35 & 24.25 & 22.45 \\
5000 words & 23.176 & 28.05 & 19.80 & 21.80 \\
10000 words & 24.118 & 25.25 & 14.90 & 14.35 \\
Terminology & 12.88 & 24.65 & 17.65 & 14.80 \\
\hline
\end{tabular}

Note: $M=$ Mean.

\subsection{Experimental Material}

120 ambiguous words were selected from previous studies (Gilhooly \& Logie 1980, Nelson et al., 1980; Durkin et al., 1989; Twilley et al., 1994; Azuma, 1996; Rodd et al., 2002) on ambiguous words. These ambiguous words are classified according to the number of words and the number of meanings in Wordsmyth online English dictionary: Words with only one entry and no less than 8 senses are polysemous words; Words with two or more entries are homonymous words. There are 60 polysemys and 60 homonyms. In view of the mental lexicon is different from the dictionary (Gernsbacher, 1984), there were 120 students from the same school, the same major and the same grade as the participants in this experiment (60 students in grade 4 and 60 students in grade 1) conducted word-meaning association test, level 5 scale assessment of word meaning familiarity, level 7 scale assessment of word meaning concreteness and level 5 subjective scale assessment of word familiarity in order to ensure that the selected ambiguous words have psychological validity of the participants. At last, 60 homonymous words and 60 polysemous words were identified as experimental ambiguous words. All ambiguous words belong to the scope of "college English teaching requirements", and at least two meanings of each word are written in the association test. In the association test, the most frequently written word meaning is the primary meaning of ambiguous words, followed by the secondary meaning. Table 2 is the index description of experimental ambiguous words.

Table 2. Index description of the experimental material

\begin{tabular}{ccccccccc}
\hline $\begin{array}{c}\text { Level of } \\
\text { L2 }\end{array}$ & $\begin{array}{c}\text { Ambiguous } \\
\text { words }\end{array}$ & $\begin{array}{c}\text { Entry } \\
(\mathrm{N})\end{array}$ & $\begin{array}{c}\text { Word } \\
\text { meaning }(\mathrm{N})\end{array}$ & $\mathrm{F}$ & $\mathrm{C}$ & $\mathrm{F}$ & $\mathrm{C}$ & Correlation \\
\hline High & $\mathrm{H}(\mathrm{M})$ & 2.22 & 2.57 & 4.58 & 4.58 & 4.28 & 4.28 & 1.85 \\
& $\mathrm{P}(\mathrm{M})$ & 1.00 & 1.42 & 4.59 & 4.59 & 4.27 & 4.27 & 4.98 \\
\hline \multirow{2}{*}{ Low } & $\mathrm{H}(\mathrm{M})$ & - & - & 4.42 & 4.42 & 4.20 & 4.20 & 2.07 \\
& $\mathrm{P}(\mathrm{M})$ & - & - & 4.44 & 4.44 & 4.29 & 4.29 & 4.97 \\
\hline
\end{tabular}

Note: $N=$ Number $F=$ Familiarity; $C=$ Concreteness; $H=$ Homonym; $P=$ Polysemy.

The analysis of the familiarity of ambiguous words revealed that the main effect of the level of the second language is significant, $\mathrm{F}(1,472)=7.454, \mathrm{p}=0.007$; The main effect of frequency of the use of meaning is also significant, $\mathrm{F}(1,472)=52.976, \mathrm{P}=0.00$. The analysis of the concreteness showed that only the main effect of second language level is significant, $\mathrm{F}(1,472)=30.079, \mathrm{P}=0.00$. 
The analysis of the correlation of ambiguous words illustrated that there is a significant difference between polysemy and homonym, $F(1,236)=693.529, p=0.00$. The results of the word meaning suggested that the differences were statistically significant between high and low-level participants, $\mathrm{F}(1,236)=21.517, \mathrm{P}=0.00$. T-test results showed that there is a significant difference of entry numbers between homonym and polysemy, $\mathrm{t}=41.329, \mathrm{p}=0.00$.

Additionally, 60 groups of true words and 120 groups of false words were selected in order to balance the experimental materials (Wang \& Sui, 2015). Each group consisted of three words, two of which are priming words and one is target word in the multiple semantic priming experiment. All three words were true in a group of true words (true word - true word - true word), and they were completely unrelated. There were two true words and a pseudoword in the group of false words. The true words were priming words and the false words were target words in the experiment. The false words were mainly selected from Previous research on ambiguous word processing (Azuma \& Orden, 1997). True words were the experimental control group, and the judgment response time of target words was collected as the baseline response time. False words were used as the fillers to ensure the judgement balance of true and false words in the experiment.

\subsection{Experimental Design}

The experiment employs level group, meaning-biased priming and semantic correlation to measure one of the factors. Level group is the variable of the participants. There are two levels: high-level group and low-level group. Meaning-biased priming is also the variable of the participants and it is divided into primary meaning-biased priming and secondary meaning-biased priming. Semantic correlation is the variable of the participants. According to the se-mantic relation between priming words and target words, it is divided into three levels: related-related (RR), unrelated-related (UR) and unrelated-unrelated (UU). When semantic relation is RR and UR, the priming words 2 are polysemous words and homonymous words, the priming words 1 and the target words are associative words of ambiguous words with two meanings. When semantic relation is UU, there is no semantic relation between priming words and target words. Table 3 is the sample of priming words and target words under all kinds of priming conditions.

Table 3. The sample of priming words and target words in the multiple semantic priming experiment

\begin{tabular}{lllll}
\hline $\begin{array}{l}\text { Priming words-semantic } \\
\text { relation of target words }\end{array}$ & $\begin{array}{l}\text { Primary meaning- biased } \\
\text { priming }\end{array}$ & $\begin{array}{l}\text { Priming words } \\
\text { (ambiguous words) }\end{array}$ & $\begin{array}{l}\text { (ambiguous words) } \\
\text { (primary meaning) }\end{array}$ \\
\hline RR & weight & fat & gas \\
UR & river & bank & save \\
UU & lion & car & apple \\
Filler & river & date & monday \\
\hline Priming words-semantic & Secondary meaning- & Priming words & 2 & target words (secendary \\
relation of target words & biased priming & (ambiguous words) & meaning) \\
\hline RR & meat & fat & weight \\
UR & save & bank & river \\
UU & apple & car & lion \\
Filler & river & date & monday \\
\hline
\end{tabular}

\subsection{Experimental Facilities and Process}

The experimental procedure was written by E-prime 2.0 software system. The presentation of experimental materials, participants' correct response time and error rate of lexical judgement were recorded. The experiment was conducted in a quiet room with 5 participants at the same time. The stimulus materials were randomly arranged and not presented repeatedly.

The experimental stimulus (in black and white) was presented in the center of a 12-inch, 1,280 $\times$ 1,289-resolution computer screen. Before the experiment, the center of the screen showed instructions for the experiment. The participants were sitting in front of the computer, with their index fingers on the computer keys of $\mathrm{F}$ and $\mathrm{J}$ respectively. Figure 1 shows the specific experimental process. It first presented a "+" fixation point in the middle of the computer screen for $500 \mathrm{~ms}$. The fixation point was then replaced by a set of masked stimulus 
symbol "\#\#\#\#\#\#". After that, the screen was blank for $500 \mathrm{~ms}$, the priming word 1 and the priming word 2 appeared successively in the position of the masked stimulus symbol. The duration was $150 \mathrm{~ms}$ respectively. Finally, the target word appeared in the position of the priming word. The participants needed to judge whether the target word is true or false. Press the key of $\mathrm{J}$ for true word and F for false word. Once the participant responded to the target word, the next "+" fixation point appeared, which is the start of the next quiz. If the participant failed to respond or made a wrong response within $3000 \mathrm{~ms}$, it would be recorded as a judgment error. Before the formal test, the participants were given a set of 30 quizzes. In the exercise, other procedures were consistent with the formal test, excepting that the participants would get feedback on whether the response was corrected or not and on the response time. There was no feedback in the formal test to determine whether the response was right or wrong and the response time. Practice results were not included in the analysis of experimental results.

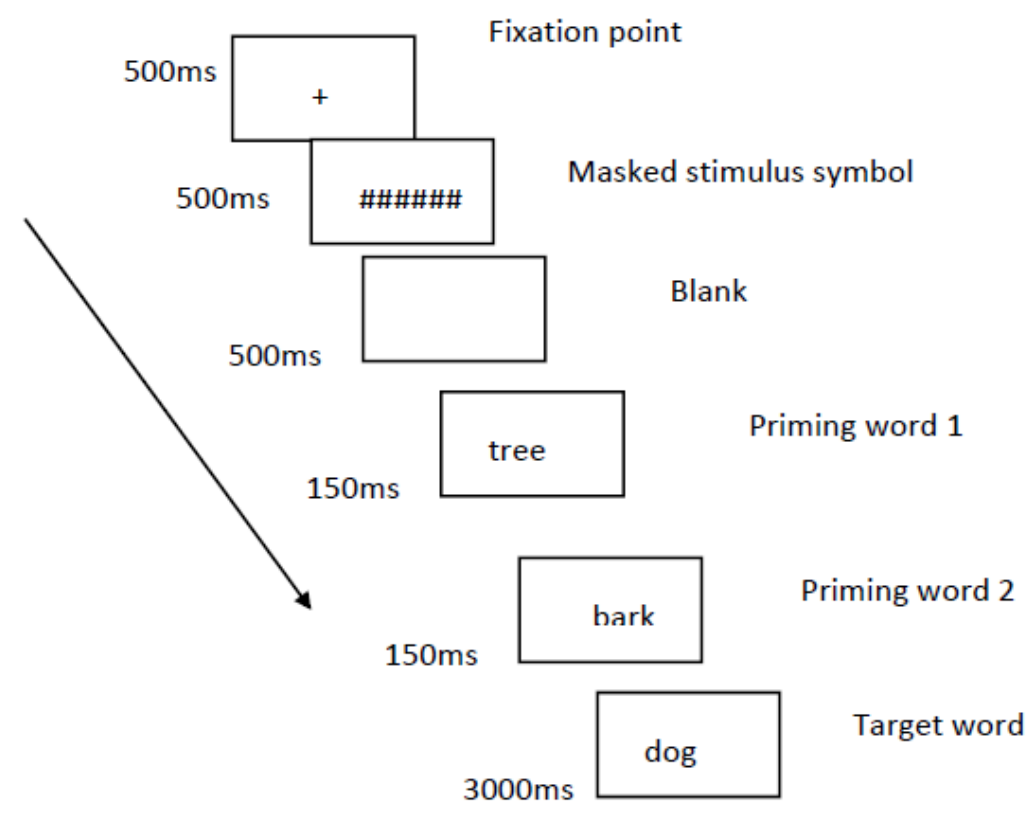

Figure 1. Procedure of the multiple semantic priming experiment

\subsection{Results and Analysis}

In the data processing, the response time less than $300 \mathrm{~ms}$ or greater than $1500 \mathrm{~ms}$ was regarded as an error, removed from the correct response time (14\%). The extreme data $(2.3 \%)$ above 3 standard deviations of the mean response time were deleted. The correct response and error rate of vocabulary judgment of the participants in high and low-level group were the indicators of experimental analysis for variance analysis. The results are presented in Table 4.

In SPSS, variance analysis of mixed design (level group $\times$ meaning-biased priming $\times$ semantic correlation) was conducted on response time and error rate of vocabulary judgment of high and low-level group under different experimental conditions. Data statistical processing included participants analysis (F1) and item (experimental material) analysis (F2).

Analysis of variance results showed that the main effect was significant in the level group, $F(1,66)=19.102$, $\mathrm{p}=0.00, \quad \mathrm{~F} 2(2,708)=29.022, \mathrm{p}=0.00$; There was significant main effect of meaning-biased priming, $\mathrm{F} 1(1,66)=8.253, \mathrm{p}=.005, \mathrm{~F} 2(1,708)=13.253, \mathrm{p}=0.00$; The main effect of semantic correlation was significant as well, $\mathrm{F} 1(1,66)=11.18, \mathrm{p}=0.01, \mathrm{~F} 2(2,708)=4.561, \mathrm{p}=0.011$. The results of pairwise comparative analysis showed that there was no significant difference between the response speed of lexical judgement of RR and UR. However, the difference was significant between the response speed of lexical judgement of UR and UU, so was the RR and UU. 
Table 4. The average response time and error rate of vocabulary judgement of different level groups of participants under various experimental conditions

\begin{tabular}{|c|c|c|c|c|c|c|}
\hline \multirow{2}{*}{$\begin{array}{l}\text { Priming words- target } \\
\text { words } \\
\text { semantic relation }\end{array}$} & & & \multirow{2}{*}{$\begin{array}{l}\text { Primary } \\
\text { priming } \\
\text { High-level }\end{array}$} & \multirow{2}{*}{$\begin{array}{c}\text { meaning-biased } \\
\text { Low-level }\end{array}$} & \multirow{2}{*}{$\begin{array}{l}\text { Secondary } \\
\text { priming } \\
\text { High-level }\end{array}$} & \multirow{2}{*}{$\begin{array}{l}\text { meaning-biased } \\
\text { Low-level }\end{array}$} \\
\hline & & & & & & \\
\hline \multirow{2}{*}{$\mathrm{RR}$} & $\begin{array}{l}\text { Average } \\
\text { Time (ms) }\end{array}$ & response & 762.14 & 871.43 & 748.91 & 784.02 \\
\hline & $\mathrm{SD}$ & & 96.57 & 134.77 & 113.60 & 118.16 \\
\hline \multirow{5}{*}{ UR } & Error rate & & $10.98 \%$ & $16.39 \%$ & $6.67 \%$ & $7.96 \%$ \\
\hline & $\begin{array}{l}\text { Average } \\
\text { Time (ms) }\end{array}$ & response & 788.41 & 826.20 & 759.32 & 801.26 \\
\hline & $\mathrm{SD}$ & & 101.27 & 140.40 & 120.04 & 133.53 \\
\hline & Error rate & & $11.27 \%$ & $18.98 \%$ & $10.29 \%$ & $13.80 \%$ \\
\hline & $\begin{array}{l}\text { Average } \\
\text { Time (ms) }\end{array}$ & response & 821.33 & 847.59 & 797.55 & 836.11 \\
\hline UU & SD & & 115.71 & 146.80 & 108.55 & 189.42 \\
\hline
\end{tabular}

The results of paired comparative analysis demonstrated that the interaction between level group and meaning-biased priming was significant, $\mathrm{F} 1(1,73)=0.006, \mathrm{p}=0.94, \mathrm{~F} 2(1,708)=52.042, \mathrm{p}=0.00$. The results of simple effect test showed that the response speed of vocabulary judgement of high-level group in the primary meaning-biased priming was significantly different from that in the secondary meaning-biased priming $(\mathrm{p}=0.00)$. Similarly, the response speed of vocabulary judgement of low-level group in the primary meaning-biased priming was significantly different from that in the secondary meaning-biased priming as well $(\mathrm{p}=0.015)$. The interaction between level group and semantic correlation was marginally significant. $F 1(1,66)=2.842, p=.062$, $\mathrm{F} 2(2,708)=0.07, \mathrm{p}=0.933$. There was a significant interaction between meaning-biased priming and semantic correlation $\mathrm{F} 1(1,73)=0.243, \mathrm{p}=0.624, \mathrm{~F} 2(2,708)=4.762, \mathrm{p}=0.009$. The results of simple effect showed that the effect of meaning-biased priming was not significant when the semantic relations between priming words and target words were RR and UR; when the semantic relationship between priming words and target words was UU, the effect of meaning-biased priming was significant $(\mathrm{p}=0.00)$.

The triple interaction of level group, meaning-biased priming and semantic relation was significant, $\mathrm{F} 1(1,66)=3.62, \mathrm{p}=0.029, \mathrm{~F} 2(2,708)=5.799, \mathrm{p}=.003$.

The results of simple effect test presented that for the high-level group, the semantic correlation effect was significant under the primary meaning-biased priming condition. $\mathrm{F}(2,132)=5.43, \mathrm{p}=0.005$; And the semantic correlation effect was significant under the secondary meaning-biased priming condition. $F(2,132)=4.05, p=0.02$. For the low-level group, the semantic correlation effect was significant under the primary meaning-biased priming condition as well, $\mathrm{F}(2,132)=3.35, \mathrm{p}=0.038$; And the semantic correlation effect was significant under the secondary meaning-biased priming condition. $F(2,132)=4.60, p=0.012$. Figure 2 and Figure 3 were respectively the profiles of the average response time of vocabulary judgement with diverse priming-target words and semantic relations of different levels of groups with various meaning biases in the experimental groups. In the high-level group, the meaning-biased effect was not significant of semantic relations in different priming-target words, while in the low-level group, the meaning-biased effect was significant only in the RR semantic relation, $F(1,67)=17.60, p=0.00$. In addition, the effect of level group was significant in the response of vocabulary judgement with the semantic relation of RR in the experimental group of primary meaning-biased priming, $F(1,67)=33.44, p=0.00$. The main effect of level group was marginal significant in the response of vocabulary judgement with the semantic relation of RR in the experimental group of secondary meaning-biased priming, $\mathrm{F}(1,67)=3.09, \mathrm{p}=.083$. The effect of level group was marginal significant in the response of vocabulary judgement with the semantic relation of UR in the experimental group of primary meaning-biased priming, $\mathrm{F}(1,67)=3.12, \mathrm{p}=.082$. Likewise, the effect of level group was marginal significant in the response of vocabulary judgement with the semantic relation of UR in the experimental group of secondary meaning-biased priming, $F(1,67)=3.56, p=0.064$. Nevertheless, the effect of level group was not significant in the response of vocabulary judgement with the semantic relation of UU in the experimental group of both primary and secondary 
meaning-biased priming, $\mathrm{F}(1,67)=2.41, \mathrm{p}=0.125, \mathrm{~F}(1,67)=1.19$, $\mathrm{p}=0.279$.

The results of error rate analysis showed that the main effect of level group was significant, $\mathrm{F}(1,66)=5.248$, $\mathrm{p}=0.025, \mathrm{~F} 2(1,708)=11.933, \mathrm{p}=0.001$. The error rate of high-level group was lower than that of low-level group. The main effect of meaning-biased priming was significant, $F 1(1,66)=6.602, p=0.012, F 2(1,708)=24.225$, $\mathrm{p}=0.00$. The error rate of vocabulary judgement with secondary meaning-biased priming $(10.50 \%)$ was lower than that with primary meaning-biased priming (14.90). The main effect of semantic correlation was significant, $\mathrm{F}(1,66)=13.224, \mathrm{p}=0.00, \mathrm{~F} 2(2,708)=7.629, \mathrm{p}=0.001$. Further analysis showed that there was a significant difference in the error rate of lexical judgement when the semantic relation was RR and UR, $p=0.00$. The error rate of lexical judgement with the semantic relation of RR $(10.50 \%)$ was significantly lower than that with the semantic relation of UR (13.60\%). There was a significant difference between the semantic relation of UR and UU (14.10\%), $\mathrm{p}=0.00$. The error rate of lexical judgement with the semantic relation of UR was significantly lower than that with the semantic relation of UU. The difference was significant between the semantic relation of $\mathrm{RR}$ and $\mathrm{UU}, \mathrm{p}=0.00$. The interaction of level group and meaning-biased priming was not significant, as well as the interaction of level group and semantic correlation. Furthermore, the triple interaction of the level, meaning-biased priming and semantic correlation was not significant.

\section{Discussion}

In terms of homonyms, the result of the experiment showed that lexical judgement response speed of high and low-level Chinese English learners in different meaning-biased priming experiments with semantic relation of UR was significantly faster than that with semantic relation of UU in multiple semantic priming, which means that there was the advantage in semantic processing of homonymous words. This result is not consistent with previous research (Gottlob et al., 1999; Rodd et al., 2002; Klepousniotou \& Baum, 2007) on the disadvantage effect of homonymous processing. According to this study, the reason for the inconsistent results is related to the masked multiple semantic priming experiment with short SOA adopted in this research. The multiple semantic priming experiment was employed by Marcel (1980) who found that there was difference of semantic processing of homonyms between masked priming and unmasked priming. Under the condition of unmasked priming with short SOA, there was no significant difference of the response speed of vocabulary judgment between the case of inconsistent semantic relations and that of the control group. The response speed of vocabulary judgement with long SOA was slower than that of the control group. However, when the semantic relation between priming words and target words is inconsistent, the response speed of vocabulary judgment is faster than that of the control group, no matter SOA is long or short under the condition of masked priming. Marcel explained the difference between the two priming conditions as follows: The participants cannot be aware of the present of priming word 1 with masked priming with short SOA. The homonym could accelerate the recognition of another related meaning-representation word. The participants would have semantic processing of homonyms under the influence of consciousness with unmasked priming. In the multiple semantic priming experiment, target words can be activated only after inhibiting the priming words when a meaning of homonym is the priming word 1 and another unrelated meaning is the target word. As the process of inhibition would take time, the judgment response speed of homonyms is slow. The participants would not stop processing their perception on the priming words though they cannot be aware of them, which is different from the unmasked priming condition. Masking only prevents the visual analysis record of the priming words from entering the consciousness of the participants. During the procedure of word processing, the pronunciation and semantics will continue to be analyzed under the condition of unconscious physical stimulation. In other words, the priming word 1 still has an impact on the semantic processing of the target words. However, the lack of visual analysis of prime word 1 saves the mapping time of morphology unit and semantic unit. After the homonymous word are activated by priming words, the other meaning of the homonymous word will be activated automatically. Thus, the judgment response time of the target word can be saved and the processing speed of homonyms is faster than that of the control group with completely unrelated semantics.

In terms of polysemous words, the result of the experiment was not consistent with the research hypothesis of the advantage in semantic processing of polysemous words of Chinese English learners. The result of the experiment illustrated that the response speed of polysemy's semantic judgement was significantly faster than that of control words among the high-level learners with the primary and secondary meaning-biased priming. Nonetheless, among the low-level learners, the response speed of polysemy's semantic judgement was faster than that of control words with the secondary meaning-biased priming and the response speed of polysemy's semantic judgement was slower than that of control words with the primary meaning-biased priming. Apparently, there is the advantage in semantic processing of polysemous words with the primary meaning, while there is disadvantage in semantic processing with the secondary meaning of the low-level learners. According to the 
theory of connectionism, there is mutual promotion and activation of semantic related meanings (McRae et al., 1997). Therefore, the disadvantage in semantic processing of polysemous words with the secondary meaning of low-level learners showed that the primary meaning has no priming impact on it, which means there is no correlation connection between two meanings. In other words, the secondary meaning representation in the mental lexicon of the low-level group has not been fully constructed, otherwise the low-level learners would have the same advantage as the high-level learners in semantic processing with both primary and secondary meaning when the semantic relation is RR. In this study, it is believed that the advantage in semantic processing of the primary meaning is the usage frequency effect of meaning. As the correlation connection between meanings is not established, the priming effect between meanings does not happen.

In addition, this study revealed that the priming effect between the meanings of polysemous words was greater than that of homonymous words. The result showed that the main effect of semantic correlation of ambiguous words was significant. Under different conditions of semantic relationship between priming words and target words, the response speed of lexical judgement is in order from fast to slow: RR, UR, UU. The error rate of judgement with semantic relationship of RR $(10.50 \%)$ was significantly lower than that of UR $(13.60 \%)$, though there was no significant difference of vocabulary judgement response speed between the semantic relationship of RR and UR. Therefore, polysemous words have more relevant semantic processing effect than homonyms. Although the correlation effect was proved in the meanings of polysemous words in this study, it also revealed that there was no inhibition effect between the meanings of homonyms with short SOA in the masked multiple semantic priming experiment. As mentioned above, this result is different from the previous research (Rodd et al. 2002) on the disadvantage effect of semantic processing of homonyms in ambiguous words processing. From this study, the main reason of inconsistent experimental result was related to experimental methods. The disadvantage in semantic processing of ambiguous words mainly occurs in conscious processing experiments. Semantic processing under conscious conditions may have strategy processing, while there was a masked semantic priming experiment with short SOA under unconscious condition, reducing the possibility of using strategy and improving automatic processing. Marcel (1980: 236), Simson and Burgess (1985: 38) once pointed out that semantic priming experiment under the condition of masking and short SOA saves the process of recording priming words by visual analysis, that is, the process of mapping from the word shape layer to the semantic layer in the mental lexicon. During the masked multiple semantic priming experiment with short SOA, the priming word 1 still had impact on semantic processing of target word though the priming word 1 was not recognized by the participants. When the homonym was activated by the priming word 1 , another meaning (the target meaning) of the homonym (the priming word 2) was activated automatically as well. In this way, the semantic processing time of target words is saved, and the processing speed is faster than that of target words under UU condition where the priming words and target words have completely unrelated meanings.

\section{Conclusion}

Through the masked multiple semantic priming experiment with short SOA, the study on semantic processing of ambiguous words showed the advantage of processing in the meaning of different frequency of use of homonymous words among Chinese English learners with high and low-level, but the advantage of processing in the meaning of different frequency of use of polysemous words does not always happen. The advantage exists in the processing of polysemous words among high-level English learners, while the advantage only exists in the meaningful processing with high frequency among low-level English learners. Furthermore, Chinese learners' semantic processing effect of polysemous words is greater than that of homonymous words. As mentioned above, the meanings can be promoted when there is an overlapping semantic feature between semantically related meanings. Although this study suggested that Chinese English learners have correlated semantic processing effect on polysemy, the questions of "whether there are overlapping semantic features between polysemous meanings; How to process overlapping semantic features; What are their representational states?" need further study.

\section{References}

Armstrong, B. C., \& Plaut, D. C. (2008). Settling Dynamics in Distributed Networks Explain Task Differences in Semantic Ambiguity Effects: Computational and Behavioral Evidence. The Proceeding of the $30^{\text {th }}$ Annual Conference of the Cognitive Science Society. https://doi.org/10.1037/e527312012-207

Armstrong, B. C., \& Plaut, D. C. (2011). Including Homonym Effects via Stimulus Quality and (Not) Nonword Difficulty: Implications for Models of Semantic Ambiguity and Word Recognition. The Proceeding of the $33^{\text {rd }}$ Annual Conference of the Cognitive Science Society.

Azuma, T. (1997). Familiarity and Relatedness of Word Meanings: Ratings for 110 Homographs. Behavior 
Research Methods, Instruments and Computers, 28. https://doi.org/10.3758/BF03203645

Beretta, A., Fiorentino, R., \& Poeppel, D. (2005). The Effects of Homonymy and Polysemy on Lexical Access: An MEG Study. Cognitive Brain Research, 24. https://doi.org/10.1016/j.cogbrainres.2004.12.006

Cruse, D. A. (1986). Lexical Semantics. Cambridge: CUP.

De Groot, A. M. B. (1984). Primed Lexical Decision: Combined Effects of the Proportion of Related Prime-target Pairs and the Stimulus-onset Asynchrony of Prime and Target. Quarterly Journal of Experimental Psychology, 36. https://doi.org/10.1080/14640748408402158

Durkin, K., \& Manning, J. (1989). Polysemy and the Subjective Lexicon: Semantic Relatedness and the Sallience of Intra- word Senses. Journal of Psycholinguistic Research, 18. https://doi.org/10.1007/ BF01067161

Gernsbacher, M. A. (1984). Resolving 20 Years of Inconsistent Interactions Between Lexical Familiarity and Orthography, Concreteness, and Polysemy. Journal of Experimental Psychology: General, 113. https://doi.org/10.1037//0096-3445.113.2.256

Gilhooly, K. J., \& Logie, R. H. (1980). Age-of-acquisition, Imagery, Concreteness, Familiarity, and Ambiguity Measures for 1944 Words. Behavior Research Methods \& Instrumentation, 4. https://doi.org/ 10.3758/BF03201693

Gottlob, L. R., Goldinger, S. D., Stone, G. O., \& van Orden, G. C. (1999). Reading Homographs: Orthographic, Phomologic, and Semantic Dynamics. Journal of Experimental Psychology: Human Perception and Performance, 2. https://doi.org/10.1037//0096-1523.25.2.561

Hino, Y., Lupker, S. J., \& Pexman, P. M. (2002). Ambiguity and Synonymy Effects in Lexical Decision, Naming, and Semantic Categorization Tasks: Interactions Between Orthography, Phonology, and Semantics. Journal of Experimental Psychology: Learning, Memory, and Cognition, 4. https://doi.org/10.1037//0278-7393.28. 4.686

Hino, Y., Kusunose, Y., \& Lupker, S. J. (2010). The Relatedness-of Meaning Effect for Ambiguous Words in Lexical-decision Tasks: When Does Relatedness Matter? Canadian Journal of Experimental Psychology, 3. https://doi.org/10.1037/a0020475

Klein, D. E., \& Murphy, G. L. (2001). The Representation of Polysemous Words. Journal of Memory and Language, 45. https://doi.org/10.1006/jmla.2001.2779

Klein, D. E., \& Murphy, G. L. (2002). Paper Has Been My Ruin: Conceptual Relations of Polysemous Senses. Journal of Memory and Language. https://doi.org/10.1016/S0749-596X(02)00020-7

Klepousniotou, E. (2007). Reconciling Linguistics and Psycholinguistics: On the Psychological Reality of Linguistic Polysemy. In R. Marina, P. Gergely, \& R. Csilla (Eds), The Cognitive Basis of Polysemy. Berlin: Perter Lang.

Klepousniotou, E., \& Baum, S. R. (2007). Disambiguating the Ambiguity Advantage Effect in Word Recognition: An Advantage for Polysemous But Not Homonymous Words. Journal of Neurolinguistics, 20. https://doi.org/10.1016/j.jneuroling.2006.02.001

Lyons, J. (1977). Semantics. Cambridge: CUP. https://doi.org/10.1017/CBO9780511620614.006

Marcel, A. J. (1980). Conscious and Preconscious Recognition of Polysemous Words: Locating the Selective Effects of Prior Verbal Context. In R. S. Nickerson (Ed.), Attention and Performance VII. Hillsdale: Erlbaum,.

McNamara, T. P. (2005). Semantic Priming: Perspectives from memory and Word Recognition. New York: Taylor \& Francis Group. https://doi.org/10.4324/9780203338001

McRae, K., de Sa, V. R., \& Seidenberg, M. S. (1997). On the Nature and Scope of Featural Representations of Word Meaning. Journal of Experimental Psychology: General, 126. https://doi.org/10.1037//0096-3445. 126.2.99

Nation, P. (1993). Vocabulary Size, Growth and Use. In R. Schreuder, \& B. Weltens (Eds.), The Bilingual Lexicon. Amsterdam: John Benjamins Publishing Company.

Needly, J. H. (1977). Semantic Priming and Retrieval from Lexical Memory: Roles of Inhibitions Spreading Activation and limited Capacity Attention. Journal of Experimental Psychology: General, 106.

Nelson, D. L., McEvoy, C. L., Walling, J. R., \& Wheeler, J. W. (1980). The University of South Florida 
Homograph Norms. Behavior Research Methods \& Instrumentation, 12. https://oi.org/10.3758/ BF03208320

Rodd, J., Gaskell, G., \& Marslen-Wilson, W. (2002). Making Sense of Semantic Ambiguity: Semantic Competition in Lexical Access. Journal of Memory and Language, 46. https://doi.org/10.1006/ jmla.2001.2810

Rodd, J. M., Gaskell, M. G., \& Marslen-Wilson, W. D. (2004). Modelling the Effects of Semantic Ambiguity in Word Recognition. Cognitive Science, 28. https://doi.org/10.1207/s15516709cog2801_4

Simson, G. B., \& Burgess, C. (1985). Actication and Selection Processes in the Recognition and Ambiguous Words. Journal of Experimental Psychology: Human Perception and Performance, 11. https://doi.org/10. 1037//0096-1523.11.1.28

Twilley, L. C., Dixon, P., Taylor, D., \& Clark, K. (1994). University of Alberta Norms of Relative Meaning Frequency for 566 Homographs. Memory \& Cognition, 22. https://doi.org/10.3758/BF03202766

Weinreich, U. (1964). Webster's Third: A Critique of Its Semantics. International Journal of American Linguistics, 30. https://doi.org/10.1086/464799

\section{Copyrights}

Copyright for this article is retained by the author(s), with first publication rights granted to the journal.

This is an open-access article distributed under the terms and conditions of the Creative Commons Attribution license (http://creativecommons.org/licenses/by/4.0/). 